\title{
Les répercussions occlusales des extractions thérapeutiques
}

\section{RÉSUMÉ}

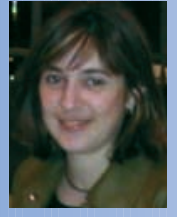

Wiam RERHRHAYE

Professeur Assistante

de l'Enseignement Supérieur

en Orthopédie Dento-Faciale,

Faculté de médecine dentaire de Rabat,

BP 6212, Rabat-Instituts, Maroc

Loubna BAHIJE

Spécialiste en Orthopédie Dento-Faciale,

Faculté de médecine dentaire de Rabat,

BP 6212, Rabat-Instituts, Maroc.

\section{Fatima ZAOUI}

Professeur de l'Enseignement Supérieur

en Orthopédie Dento-Faciale

Faculté de médecine dentaire de Rabat,

BP 6212, Rabat-Instituts, Maroc.

\section{El Housseine AALLOULA}

Professeur de l'Enseignement Supérieur

et chef du service

d'Orthopédie Dento-Faciale,

Faculté de médecine dentaire de Rabat,

BP 6212, Rabat-Instituts, Maroc.

En tant qu'orthodontistes, nous sommes souvent appelés à pratiquer des extractions pour répondre à nos objectifs thérapeutiques, esthétiques, fonctionnels, occlusaux et dento-squelettiques. Les conséquences occlusales, statiques et dynamiques, de ces extractions ne sont pas toujours anodines, et certains gestes complémentaires sont alors nécessaires lors de la phase de finition orthodontique afin d'assurer la stabilité de nos traitements.

Nous devons donc être "occluso-conscients» pour minimiser ces inconvénients et obtenir un équilibre occlusal optimal.

À travers cet article, nous essayerons de passer en revue toutes les indications d'extractions prévues par le plan de traitement orthodontique, des prémolaires aux molaires, en passant par les incisives et ce tout en abordant les conséquences statiques et dynamiques sur l'occlusion ainsi que les moyens d'y remédier.

- extractions

- orthodontie

occlusion
AOS 2008:243:239-248

DOI:10.1051/AOS:2008037

(C) AEOS / EDP Sciences 


\section{Introduction}

$>$

L'extraction de dents adultes est sou-

vent envisagée dans le cadre d'un traitement orthodontique afin de résoudre au mieux un problème d'encombrement, de proalvéolie incisive ou de décalage occlusal. Cependant, cette décision thérapeutique doit prendre en considération les conséquences de la perte de ces dents. En effet, l'avulsion d'élé- ments dentaires et la fermeture des espaces d'extraction s'accompagnent obligatoirement de modifications majeures au niveau de l'agencement dentaire intra-arcade et interarcade. Ainsi, Dawson conseille d'envisager les extractions avec la plus grande prudence, notamment dans le cas d'occlusion initiale stable au niveau des groupes cuspidés[1].

\section{Impératifs occlusaux du traitement orthodontique}

En fin de traitement orthodontique il faut chercher à obtenir une position d'équilibre occlusal servant de contention naturelle. Toute inocclusion est un facteur d'instabilité et de récidive[2]. Bien que d'autres facteurs puissent entraîner une récidive, la prévenir c'est avant toute chose maintenir les acquis occlusaux.

Pendant la finition de son traitement, l'orthodontiste doit s'inquiéter de la direction des axes dentaires car sans une position précise et correcte des racines, I'alignement coronaire ne restera pas stable[3].

De plus, les rapports inter-arcades sont primordiaux pour la stabilité des traitements orthodontiques et dépendent en grande partie de I'harmonie des rapports intra-arcades.

\section{Les rapports intra-arcades}

Les cuspides vestibulaires et les bords libres, les cuspides linguales et les sillons mésio-distaux doivent s'inscrire dans des courbes harmonieuses et sensiblement parallèles (fig. 1).

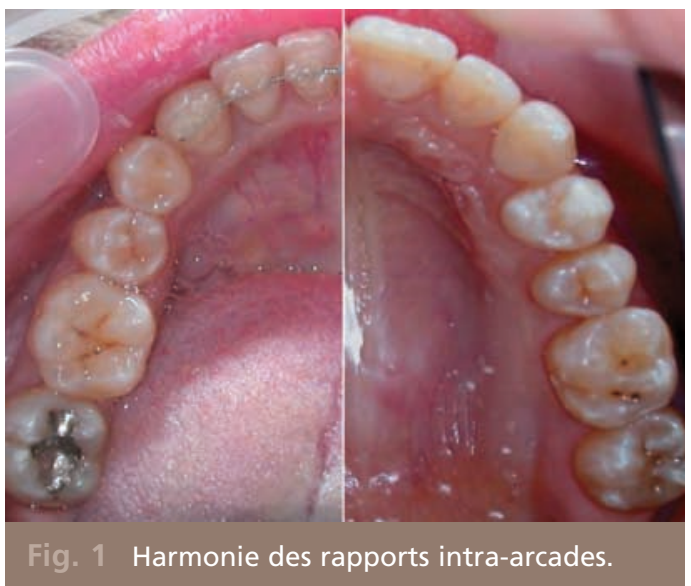

Les six clés de l'occlusion normale[4] contribuent, ensemble ou séparément, à la stabilité de l'occlusion et sont considérées par plusieurs auteurs comme essentielles au succès d'un traitement orthodontique. Ces clés se résument comme suit :

- le pan distal de la cuspide disto-palatine de la première molaire supérieure s'engrène avec le pan mésial de la cuspide mésio-lin- 

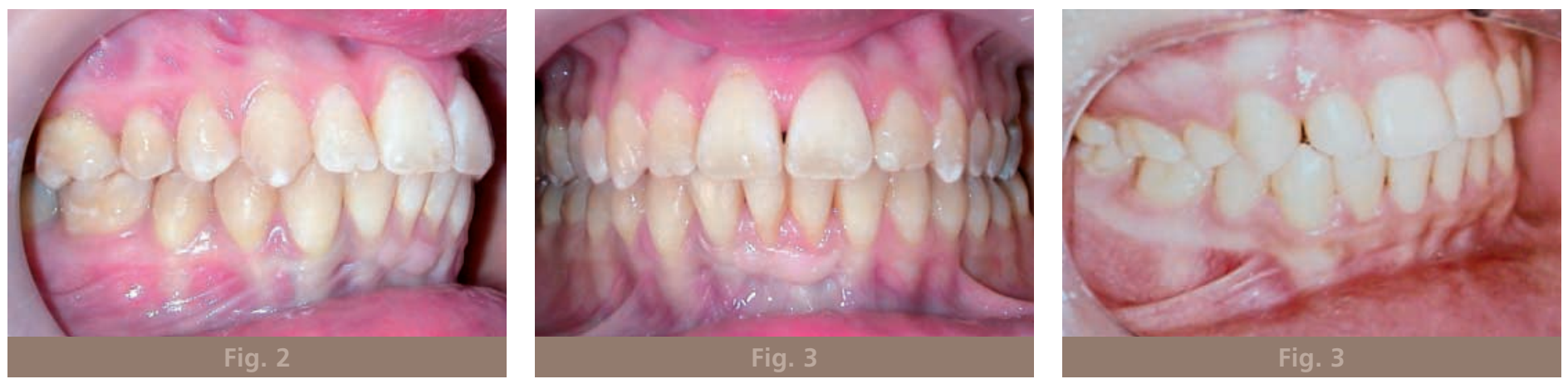

Fig. 2 à 4 Les six clés de l'occlusion selon Andrews.

guale de la seconde molaire inférieure (fig. 2) ;

- les grands axes verticaux coronaires des dents sont inclinés en direction mésio-occlusale. La partie gingivale de la couronne doit être plus distale que la partie occlusale. Le degré de version de ces dents détermine l'espace mésio-distal qu'elles occupent. Cette version a donc une influence considérable sur l'occlusion (fig. 2 et 3 ) ;

- I'inclinaison vestibulaire de la couronne des incisives a une répercussion sur l'engrènement molaire ; des incisives trop droites sont accompagnées de canines et prémolaires trop mésiales ou de diastèmes, que l'on met à tort sur le compte de la dysharmonie dento-dentaire (DDD) ;

- les surfaces coronaires des faces vestibulaires sont inclinées en haut et en dedans à la mandibule, en bas et en dehors au maxillaire ;

- il ne doit y avoir aucune rotation, les crêtes marginales doivent être à la même hauteur et les contacts interproximaux serrés en absence de DDD. S'il y a DDD, il ne faut pas chercher à fermer les diastèmes aux dépens d'une bonne occlusion (fig. 4) ;

- enfin, le plan d'occlusion doit être plat pour une intercuspidation maximale.

\section{Les rapports inter-arcades}

L'occlusion doit répondre idéalement à un certain nombre de critères[5] :

\section{- Dans le sens antéro-postérieur}

(fig. 2)

La Classe I d'Angle se manifeste par la mésioposition d'une demi cuspide des dents mandibulaires par rapport aux dents maxillaires. La Classe canine est plus importante, cependant la première molaire est qualifiée de «clé d'occlusion» et sa cuspide mésio-vestibulaire est reçue dan le sillon vestibulaire de la première molaire inférieure. D'après Ricketts: «une superbe occlusion se caractérise par le contact entre le versant distal de la seconde prémolaire supérieure et la face mésiale de la première molaire inférieure»[6].

\section{- Dans le sens transversal}

(fig. 3)

L'arcade supérieure doit circonscrire l'arcade inférieure de la valeur d'une cuspide. Les lignes inter incisives doivent coïncider entre elles et avec la ligne sagittale médiane.

\section{- Dans le sens vertical}

(fig. 4)

Les dents maxillaires doivent recouvrir les dents mandibulaires de $2 \mathrm{~mm}$ au niveau anté- 
rieur, ce recouvrement diminuant de mésial en distal. Les rapports dento-dentaires doivent se faire selon le mode cuspide/fosse (au niveau molaire), cuspide/embrasure (au niveau prémolaire) et bord libre/crête marginale (au niveau antérieur).

Enfin, en orthodontie, il faut rechercher une coïncidence entre la relation centrée et l'intercuspidie maximale sans aucun dérapage antéro-postérieur. Un décalage amène une surcharge incisive responsable, entre autres, de récidive du diastème interincisif[7].

La position physiologique d'intercuspidation doit s'établir avec des contacts punctiformes, de même intensité et répartis symétriquement sur l'arcade. Cette répartition permet une activité musculaire équilibrée. Lors de traitements comportant des extractions, il est donc important d'observer les répercussions éventuelles de ces extractions sur l'agencement des contacts occlusaux.

\section{L'occlusion dynamique[5]}

La dynamique mandibulaire doit répondre à deux exigences fondamentales :

\section{- En propulsion}

On doit obtenir une désocclusion postérieure grâce au guide antérieur. Pour ce, le guide antérieur doit augmenter avec la hauteur cuspidienne. La qualité du guide antérieur en matière de désocclusion postérieure sera proportionnelle à la valeur de la pente incisive, et donc sera influencée par le recouvrement et le surplomb qui doivent être de $2 \mathrm{~mm}$ (fig. 4).

\section{- En latéralité}

Il faut obtenir une désocclusion de toutes les dents du côté non travaillant. Deux schémas fonctionnels peuvent être observés : une fonction canine où seule la canine sert de guide du côté travaillant, ou une fonction de groupe assurée par la canine associée aux cuspides vestibulaires des prémolaires et mésio-vestibulaires des premières molaires supérieures.

\section{Cas particulier des extractions orthodontiques}

Devant la diversité des situations cliniques que rencontre le praticien, certains cas vont nécessiter une individualisation des objectifs de finition en adoptant des compromis occlusaux. Les extractions orthodontiques peuvent créer une DDD ne permettant pas une relation occlusale optimale. L'orthodontiste, dans ce cas, ne doit pas fermer les diastèmes aux dépens d'une bonne occlusion. Les diastèmes persistants seront comblés par l'accentuation des courbures de deuxième ordre (pour occuper plus d'espace mésio-distal), par l'apport de matériaux composites ou par des facettes céra- miques ou des prothèses. La persistance de ces diastèmes peut compromettre la stabilité du traitement du fait de l'absence de points de contacts proximaux (fig. 4).

\section{Extractions symétriques et homologues : 14-24-34-44 ou 15-25-35-45[8, 9, 10]}

On vise l'obtention, en fin de traitement, d'une classe I molaire et canine avec réduction 


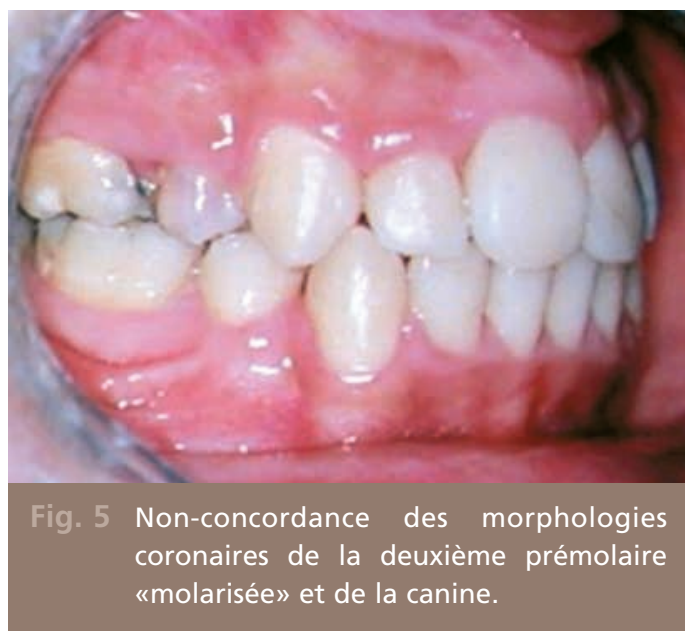

de la longueur d'arcade. On peut observer des problèmes de contacts inter-proximaux par non-concordance des morphologies coronaires (la deuxième prémolaire «molarisée» est en contact avec une canine) (fig. 5).

\section{Extractions symétriques mono maxillaires : $14-24$ ou $15-25$}

À défaut d'apparaître comme une solution thérapeutique idéale, elles représentent un compromis utile et simple.

Cependant, on se trouve face à une situation de classe II thérapeutique qui pose un problème d'interférence du côté travaillant, en latéralité[11] (fig. 6). Cette solution présente un risque d'usure, certes, mais permet de préserver le capital dentaire.

\section{> Équilibre statique}

\section{- Conséquences intra-arcades[11]}

- Perte de la continuité des formes entre la canine et la deuxième prémolaire maxillaire.

- Perte de continuité des crêtes entre la première molaire et la première prémolaire maxillaire.

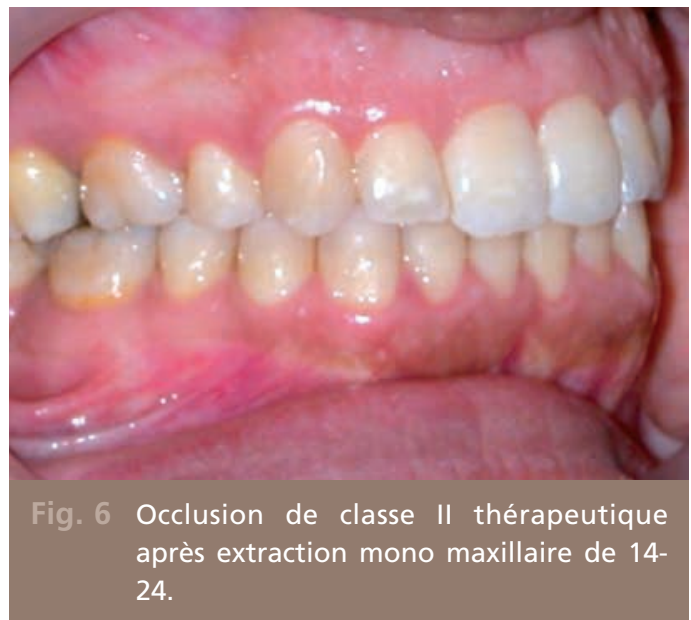

- Risque de réouverture des espaces d'extraction.

- Conséquences inter-arcades[5, 11]

- Stabilité amoindrie par réduction du nombre de points de contact et par leur disposition défavorable.

- Peu de modifications au niveau des prémolaires.

- Perte de l'ensemble des verrous de centré au niveau molaire (perte du contact tripodique). Les doubles rapports cuspides-fosses centrales sont remplacés par des rapports cuspides-embrasures ou fosses marginales (contacts dipodiques).

- Mauvaise coordination sagittale et transversale des arcades.

- En l'absence de la troisième molaire maxillaire, la cuspide disto-vestibulaire de la deuxième molaire mandibulaire perd son antagoniste.

\section{> Équilibre cinétique[11]}

\section{- En propulsion}

Pas d'interférences.

\section{- En latéralité travaillante}

L'échappement cuspidien est gêné par le fait que la cuspide mésio-vestibulaire de la pre- 
mière molaire mandibulaire se trouve face au pont d'émail.

\section{- En latéralité non travaillante}

Incoordination morphologique cuspides/structures de dégagement. C'est donc une occlusion instable avec affrontement de structures convexes et augmentation du risque d'usure prématurée des dents.

\section{$>$ Conduite à tenir}

Dans ce type d'occlusion, l'importance de la cuspide vestibulaire de la première prémolaire mandibulaire peut nécessiter l'aménagement d'une logette par améloplastie au niveau de la fosse marginale de la seconde prémolaire maxillaire[8].

Dans ces traitements, le praticien ne doit pas provoquer de rotation disto-vestibulaire au niveau des premières molaires maxillaires (rotation que l'on peut rechercher dans le cas d'extractions bimaxillaires) pour pouvoir mettre en contact la cuspide disto-palatine de la première molaire maxillaire avec la fosse mésiale de la première molaire mandibulaire[8].

Une fonction canine permettra une désocclusion postérieure immédiate[11].

\section{Extraction des molaires : $36-46[1,6]$}

\section{> Conséquences statiques}

- DDD par excès maxillaire ;

- obtention d'une classe I canine et d'une pseudo classe I molaire ;

- les racines de la deuxième molaire mandibulaire sont convergentes, offrant moins de résistance aux forces occlusales au niveau de leur embrasure que les premières molaires;
- s'il existe un retard d'évolution de la troisième molaire mandibulaire, la deuxième molaire maxillaire restera quelques temps sans antagoniste ;

- obligation d'extraction des troisièmes molaires maxillaires ;

- la répartition des charges occlusales est moins bonne car la cuspide mésio-palatine de la première molaire maxillaire s'engrène dans la fosse marginale distale de la deuxième molaire mandibulaire.

\section{> Conséquences cinétiques}

- dégagement moins facile de la cuspide mésio-palatine de la première molaire maxillaire en latéralité non travaillante, du fait de la non-concordance des faces occlusales de la première molaire maxillaire (en $\mathrm{Y}$ ) et celle la deuxième molaire mandibulaire (cruciforme) ;

- la cuspide d'appui mésio-palatine de la première molaire maxillaire est distalée par rapport à la fosse centrale de la deuxième molaire mandibulaire ce qui peut entraîner une interférence en latéralité travaillante ;

- les risques d'usure sont augmentés reflétant les difficultés d'engrènement et de mouvement car la cuspide mésio-vestibulaire de la première molaire maxillaire crée une fosse plus large au niveau de la face occlusale de la deuxième molaire mandibulaire. De plus le pont d'émail est en rapport avec une structure convexe d'où l'instabilité du contact ;

- risque d'interférence en latéralité non travaillante de la cuspide mésio-palatine de la première molaire maxillaire avec les cuspides d'appui mandibulaires.

\section{$>$ Conduite à tenir}

- pour améliorer ce système, on peut créer des diastèmes entre la seconde molaire et la 
deuxième prémolaire mandibulaires et la troisième et la seconde molaires maxillaires ;

- une coronoplastie par meulage sélectif peut minimiser le problème.

\section{Extraction des molaires :} $16-26[1,6]$

On cherche l'obtention d'une classe I canine et une pseudo classe I molaire mais cette dernière présente une situation plus avantageuse car elle va dans le sens d'une DDD par excès mandibulaire. La DDD est due à la diminution de la hauteur et du diamètre coronaires et radiculaires ( $2^{\mathrm{e}}$ molaire $<1$ re molaire) et à la tendance à la fusion des racines.

\section{> Conséquences intra-arcades}

- la deuxième molaire maxillaire présente une convergence des faces proximales s'accompagnant d'un décalage distal de la cuspide mésio-palatine :

- la cuspide disto-palatine est presque inexistante.

\section{> Conséquences inter-arcades}

- la cuspide centro-vestibulaire de la première molaire mandibulaire est rarement calée dans la fosse centrale de la deuxième molaire maxillaire qui est déportée mésialement ;

- en général la troisième molaire maxillaire est trop courte, avec peu d'acuité cuspidienne et aura une relation de tangente au niveau de la deuxième molaire mandibulaire.

\section{Extraction des molaires : $16-26-36-46[6]$}

Ce système est moins générateur d'interférences occlusales mais présente une importante instabilité. L'usure est moins marquée et on observe souvent un diastème entre secondes prémolaires et secondes molaires maxillaires.

\section{Extraction}

d'une incisive mandibulaire chez l'adulte (fig. 7)

\section{$>$ Indications $[6,12]$}

- orthodontie préprothétique : encombrement incisif mandibulaire gênant la réalisation d'un bridge supérieur ;

- orthodontie parodontale ;

- souci esthétique: dans le cas d'un profil concave, on peut hésiter à extraire, même des secondes prémolaires afin de ne pas modifier la position des lèvres ;

- classe I parfaite avec encombrement incisif mandibulaire (DDD).

\section{$>$ Avantages $[6,13]$}

- diminution de la durée du traitement ;

- préservation du parodonte car cette solution engendre peu de mouvements dentaires ;

- I'appareillage multi-attache peut se limiter à I'arcade mandibulaire voire à I'arc incisivocanin inférieur.

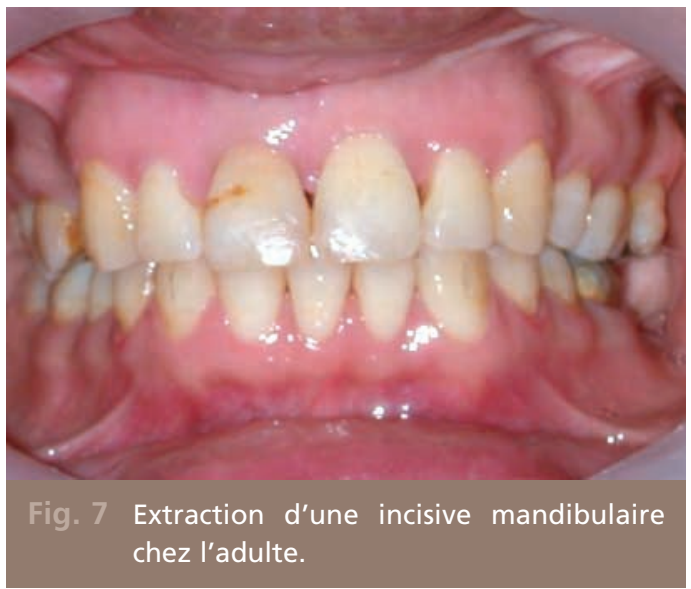


> Inconvénients[6, 14, 15]

Création d'une DDD antérieure par excès maxillaire entraînant un surplomb d'où un guide antérieur réduit.

\section{$>$ Conduite à tenir}

- le surplomb antérieur peut être diminué par réduction amélaire au niveau des incisives maxillaires ;

- les rapports inter canins dépendent de deux facteurs plus ou moins liés : le choix de l'incisive extraite (centrale ou latérale) et la position finale du milieu interincisif maxillaire par rapport au centre de la face vestibulaire de l'incisive mandibulaire devenue médiane ;

- une contention prolongée de l'arc incisif mandibulaire est nécessaire car il y a peu de recul en ce qui concerne la stabilité de ce type de traitement[16]. Néanmoins, on peut noter une meilleure stabilité des cas traités par extraction d'incisive inférieure[17, 18, 19].

\section{Agénésie des incisives latérales supérieures avec fermeture des espaces[1, 6, 20]}

La substitution des canines aux incisives latérales crée des rapports de classe II canine avec perte de la fonction canine. On opte alors pour une fonction de groupe.

\section{> Conséquences intra-arcades}

La coronoplastie canine permet un contact avec la face distale de l'incisive centrale et la face mésiale de la première prémolaire, cependant ce remodelage n'établit pas des rapports interproximaux de qualité : risque de réouverture d'espace, bourrage alimentaire...

\section{> Conséquences inter-arcades}

Les inconvénients de la classe II canine viennent s'ajouter à ceux de la classe II thérapeutique.D'autre part, le guide antérieur est affecté par la morphologie des canines qui est différente de celle des incisives latérales.

\section{$>$ Conduite à tenir}

La perte de la fonction canine peut être compensée soit par :

- la création d'une nouvelle fonction canine par la première prémolaire. II faut alors réaliser un torque différentiel radiculo-palatin et une coronoplastie par soustraction au niveau de la face palatine et/ou vestibulaire et du bord libre des canines. Ce remodelage coronaire répond à des impératifs esthétiques et surtout occlusaux afin d'assurer la stabilité des résultats ;

- la création d'une fonction de groupe.

Un set-up dynamique sur articulateur semiadaptable précise avant la fin du traitement orthodontique les mouvements dentaires nécessaires.

\section{Extraction asymétrique d'une prémolaire[21, 22, 23]}

Elle est indiquée si la prémolaire concernée est incluse, agénésiée, encombrée ou présentant des dysplasies amélaires. On obtient une classe I canine et molaire d'un côté et une classe II ou III molaires du côté de l'extraction maxillaire ou mandibulaire.

Elles nécessitent une finition du cas par hémiarcade sans pour autant nuire à l'harmonie occlusale de l'ensemble (fig. 8). En général et dans la mesure du possible, il vaut mieux éviter les extractions asymétriques. 


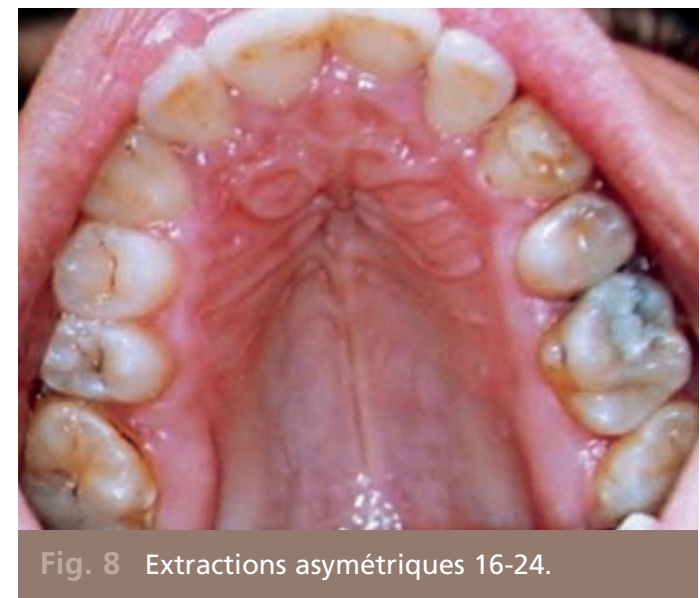

\section{Conclusion}

Nos traitements offrent des chances de rééquilibration face à des arcades en malposition, dysharmoniques ou mutilées par des lésions carieuses. Bien que notre but soit la conservation et l'harmonisation de toutes les dents, il arrive que des extractions soient nécessaires. De plus, quand le succès occlusal n'est pas

\section{Extractions symétriques} et non homologues : 14-15-34-44 ou 14-24-34-45[10]

Il y a un problème de continuité d'arcade et de contacts interproximaux : les premières prémolaires maxillaire et mandibulaire sont plus caniniformes que les deuxièmes prémolaires, ceci est encore plus accentué à l'arcade mandibulaire.

total, n'est-il pas préférable à ce qui existait avant. Nous devons être "occluso-conscients» selon les critères de l'occlusion fonctionnelle, occlusion qui aboutit à un état harmonieux de tolérance mutuelle des différents constituants de l'appareil manducateur.

\section{Bibliographie}

1. Dawson PE.

Les problèmes de l'occlusion clinique: évaluation, diagnostic et traitement. Paris, Cdp 1992:582p

2. Boley JC. Long-term stability of Class I premolar extraction treatment. Am J Orthod Dentofacial Orthop 2003;124:277-287.

3. Huggins D. The retention phase of treatment: the importance of root positioning as an aid to stability of the occlusion. Austr Orthod J 1994;13: 100-105.
4. Andrews LF.

The six keys to normal occlusion. Am J Orthod 1972;62:296-309.

5. Slavicek $R$ et coll. Les concepts de l'occlusion. Rev Orthop Dent Fac 1983;17:449-490.

6. Bassigny $\mathrm{F}$. Manuel d'Orthopédie Dento-Faciale. Masson, Ed Paris, 1986.

7. Jolivet $\mathrm{C}$ Argumentation du rapport. Orthod Fr 1985:56:327-341.

8. Bartala M, Boileau MJ. Conséquences occlusales et articulaires des extractions

de prémolaires :

revue de la littérature.

Rev Orthop Dento-Fac 2001;35:223-243.

9. Tweed $\mathrm{Ch}$. Clinical orthodontics.

Vol. 1. St. Louis : Mosby, 1966

10. Weirich TP

Les cas orthodontiques limites entre extraction et non-extraction de prémolaires. Inter Orthod 2003;1:63-94.

11. Lejoyeux $\mathrm{E}$. L'occlusion thérapeutique de classe II molaire. 
Rev Orthop Dent Fac 1983;17:549-568.

12. Kokich VG, Shapiro PA. Lower incisor extraction in orthodontic treatment. Angle Orthod 1984;54:139-153.

13. Kokich Vo.

Treatment of a Class I malocclusion with a carious mandibular incisor and no Bolton discrepancy. Am J Orthod Dentofacial Orthop 2000;118:107-113.

14. Bolton WA. Disharmony in tooth size and its relation to the analysis and treatment of malocclusion. Angle Orthod 1958;28:113-130.

15. Gilmore CA, Little RM. Mandibular incisor dimensions and crowding. Am J Orthod 1984;85:493-563.

16. Riedel RA \& al. Mandibular incisor extraction: Postretention evaluation of stability and relapse.

Angle orthod 1992;62:103-116.

17. Blake M, Bibby $K$. Retention and stability: a review of the literature. Am J Orthod Dentofacial Orthop 1998;114:299-306.

18. Le Gall M.

Rapport de la récidive. Orthod Fr 1999;70:15-137.

19. Little RM, Wallen $T R$, Riedel RA. Stability and relapse of mandibular anterior alignment - first premolar extraction cases treated by traditional edgewise orthodontics.

Am J Orthod 1981;80:349-365.

20. Canal P, Chabre $C$.

Agénésie des incisives

latérales supérieures : Quel traitement ?
Orthod Fr 1987:58:265-361.

21. Janson $\mathrm{G}$

Class II subdivision

treatment success rate with symmetric and asymmetric extraction protocols. Am J Orthod Dentofacial Orthop 2003;124:257-264.

22. Schacter RI. Treatment of an adult patient with severely crowded bimaxillary protrusive Class II malocclusion with atypical extractions.

Am J Orthod Dentofacial Orthop 2002;122:317-322.

23. Shelley A.

Asymmetric extraction treatment of an Angle Class II Division 2 subdivision left malocclusion with anterior and posterior crossbites.

Am J Orthod Dentofacial Orthop 2000;118:462-466.

\section{SUMMARY}

\section{Occlusal repercussions of therapeutical extraction}

Wiam RERHRHAYE

Loubna BAHIJE

Fatima ZAOUI

El Housseine AALLOULA

Keywords

- extractions

- orthodontics

- occlusion
Orthodontists often practice extractions to reach aesthetic, functional, occlusal and dentosquelettal goals of treatment. The occlusal consequences of those extractions are not harmless, and necessite suplemantary precautions when finishing to garantie stability of results.

We must be "occlusoconscient" to minimise inconvenients et obtaine an optimal occlusal balance. 